\title{
$\mathrm{L}$ - arginine acetate single crystals for NLO applications
}

\author{
P. Gnanasekaran ${ }^{\mathrm{a}}$, and J. Madhavan ${ }^{\mathrm{b}^{*}}$ \\ ${ }^{a}$ Department of Physics, C. Kandaswami Naidu College, Chennai - 600 102, India \\ ${ }^{b}$ Department of Physics, Loyola College, Chennai - 600034 \\ jmadhavang@yahoo.com
}

\begin{abstract}
Single crystals of pure and Lanthanum doped L- arginine acetate (LAA) were grown successfully by slow evaporation technique. The grown crystals were confirmed by X-ray powder diffraction studies. The pure and doped crystals were characterized by Fourier Transform Infrared (FT-IR) and UV-Vis-NIR studies. It was found that the optical properties were enhanced by the incorporation of the dopant. Nonlinear optical studies reveal that the dopant has increased the efficiency of the LAA crystal.
\end{abstract}

Keywords: L-arginine acetate crystal, dopant, lanthanum, NLO.

Introduction

L-arginine acetate (LAA) is one of the prominent members of the L-arginine phosphate (LAP) family (Monaco et al., 1987) identified as the most promising organic nonlinear material. Recent studies reveal that LAA possess excellent optical, thermal, mechanical properties which make it a strong candidate for photonic devices (Pal \& Kar, 2005). The present work deals with the influence of rare earth dopant (Lanthanum) on the growth and characterization of LAA single crystals.

Experimental procedures

From aqueous solution with equimolar proportion of L-arginine (kemphasol 98\%) and acetic acid (AR grade) $\mathrm{LACH}_{3} \mathrm{COOH}$ is formed by slow evaporation technique. The $\mathrm{pH}$ values of LAA and La-LAA were 6.0, 6.5 respectively. The concentration of the dopant was maintained at $2 \%$. Optically clear LAA and La-LAA were harvested after a period of 35 days. The grown crystals were subjected to powder X-ray diffraction to confirm the crystallinity and also to estimate the lattice parameters. The FT-IR spectra were taken using the $\mathrm{KBr}$ pellet technique to confirm the presence of functional groups. UV-Vis-NIR absorption studies were also done and the spectrums were recorded. The SHG efficiencies were studied using a $Q$ switched, mode locked Nd:YAG laser.

Results and discussion

Powder X-ray diffraction analysis

Fig. 1 and Fig. 2 show the powder X-ray diffraction pattern of pure and doped crystals

Table1. Crystal data for $\angle A A$ and $\angle a-L A A$

\begin{tabular}{|l|c|c|c|c|c|}
\hline Sample & $\mathrm{a}(\AA)$ & $\mathrm{b}(\AA)$ & $\mathrm{c}(\AA)$ & $\beta^{\circ}$ & $\begin{array}{c}\text { Volume } \\
\AA^{3}\end{array}$ \\
\hline LAA & 9.221 & 5.184 & 13.090 & 109.61 & 586.20 \\
La-LAA & 9.114 & 5.232 & 12.794 & 107.66 & 581.38 \\
\hline
\end{tabular}

respectively. From the X-ray diffraction data, it is observed that both pure and doped crystals crystallize in the monoclinic system with space group $\mathrm{P} 2{ }_{1}$ (Muralidharan et al., 2003). The crystal data of LAA and La-LAA are presented in Table.1 Inductively coupled plasma analysis

The exact percentages of dopants present in the crystal lattice were determined by Inductive Coupled Plasma (ICP) analysis. The result shows that $15 \mathrm{ppm}$ of Lanthanum entered into the crystal lattice of the crystal. It is seen that the amount of dopant incorporated in to the doped crystal is less than the concentration of the dopant in the corresponding solution.

FT-IR analysis

The middle infrared spectrum of pure and doped LAA is shown in Fig. 3 and Fig. 4 respectively. The spectra show a broad envelope between 2100 and $3500 \mathrm{~cm}^{-1}$. It is due to overlapping of peaks of $\mathrm{NH}\left(\mathrm{NH}_{3}{ }^{+}\right)$vibrations, $\mathrm{OH}(\mathrm{COOH})$ and $\mathrm{CH}\left(\mathrm{CH}_{2} \& \mathrm{CH}_{3}\right)$. The $\mathrm{C}=\mathrm{O}$ stretch of $\mathrm{COO}^{-}$gives its peak at $1689 \mathrm{~cm}^{-1}$ whereas the asymmetric $\mathrm{NH}$ bend of $\mathrm{NH}_{3}{ }^{+}$shows its peak at $1639 \mathrm{~cm}^{-1}$. The corresponding symmetrical $\mathrm{NH}$ bend is assigned to the peak at $1532 \mathrm{~cm}^{-1}$. All these peaks overlap and produce broad intense envelope. In this envelope, the peak due to asymmetric $\mathrm{CO}_{2}$ stretch is observed at $1400 \mathrm{~cm}^{-1}$. All the other peaks below $1400 \mathrm{~cm}^{-1}$ are due to $\mathrm{COO}^{-}$and other bending modes. The torsional $\mathrm{NH}$ oscillation of $\mathrm{NH}_{3}{ }^{+}$gives its peak at $543 \mathrm{~cm}^{-1}$. From the results obtained form FT-IR spectrum it is concluded that the acetic acid proton is not transformed $-\mathrm{COO}^{-}$group of $\mathrm{L}$-arginine, but it its one of the unprotanated amino group.

\section{UV-Vis-NIR absorption studies}

The absorption spectra are shown in Fig. 4. From the absorption spectra it was seen that the absorption is minimum in the entire visible region for both pure and doped crystals. Though the lower cut-off for both pure and doped crystals were found to be around $240 \mathrm{~nm}$, the lower percentage of absorption for doped one in comparison to pure LAA is likely to improve the nonlinear optical (NLO) property.

\section{Second Harmonic Generation studies}

The results obtained from SHG studies (Kurtz \& Perry, 1968) reveal that the efficiency of frequency doubling in LAA $(24.6 \%)$ and Mg-LAA $(33.8 \%)$ were better than KDP $(08 \%)$. It was 
Fig. 1. Powder $X$-ray diffraction pattern of $L A A$; Fig.2. Powder $X$-ray diffraction pattern of $L a-L A A$; Fig.3. FT-IR spectrum of pure $L A A$; Fig. 4. FT-IR spectrum of $L a-L A A$.
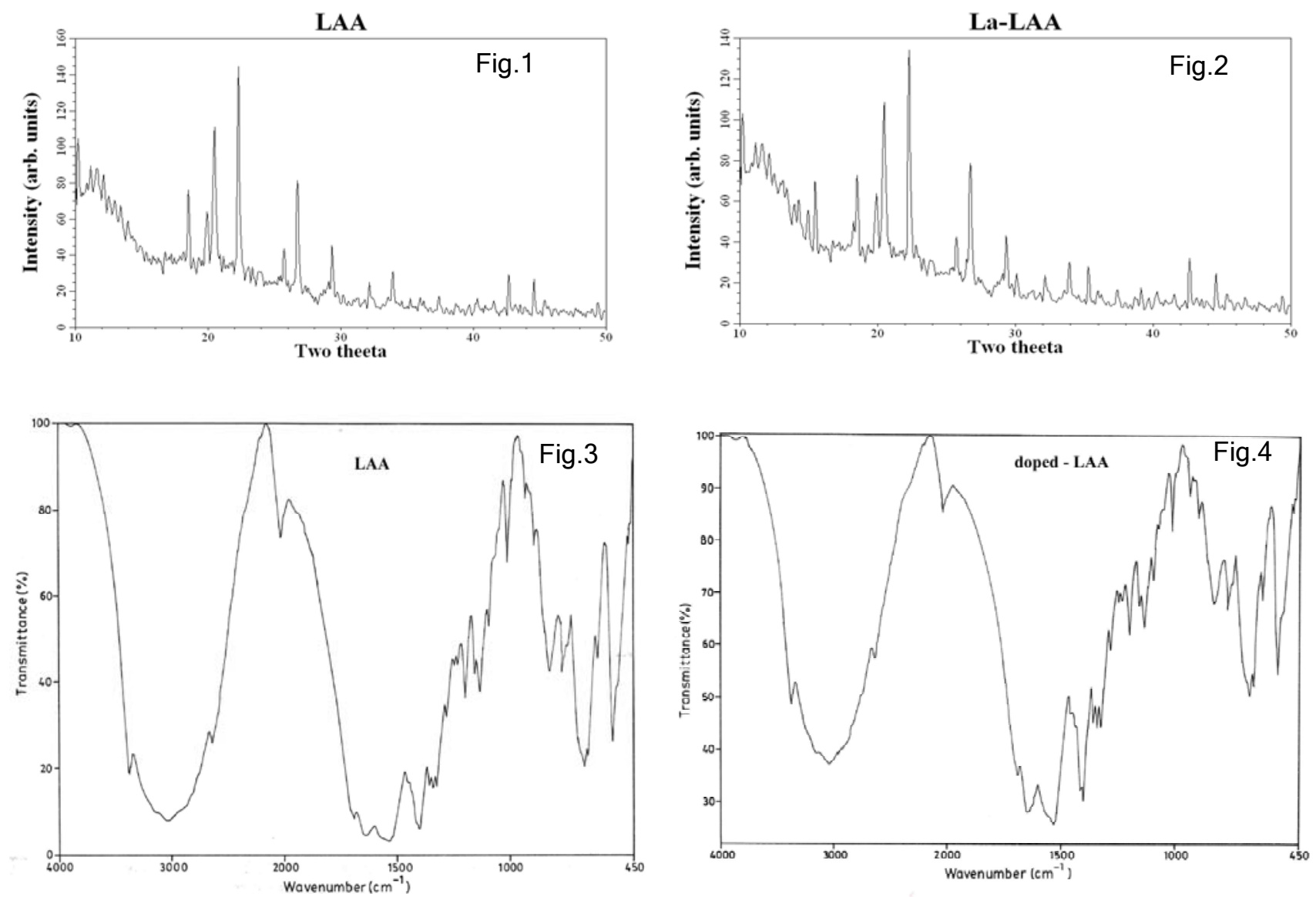

also found that La doped crystal has an efficiency equivalent to that of LAP (33\%) for the same input power.

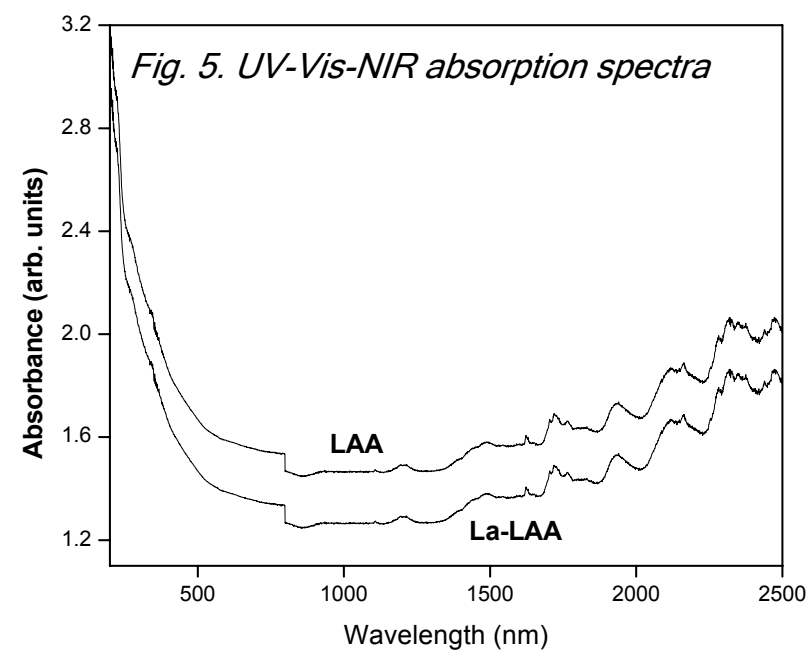

Conclusion

The studies confirm that the grown crystals were non-linear in nature and the metal substitution has enhanced the non-linearity of the crystals.

Reference

1. Kurtz SK and Perry TT (1968) A Powder technique for the evaluation of nonlinear optical materials. J. Appl. Phys. 39, 3798-3813.

2. Monaco SB, Davis LE, Velsko SP, Wang FT and Eimerl D (1987) Synthesis and characterization of chemical analogs of Larginine phosphate. J. Crystal Growth. 85, 252-255.

3. Muralidharan R, Mohankumar R, Jayavel R and Ramasamy P (2003) Growth and characterization of L-arginine acetate single crystals: a new NLO material. J. Crystal Growth. 259, 321-325.

4. Pal T and Kar T (2005) Optical, mechanical and thermal studies of nonlinear optical crystal L-arginine acetate. Materials Chemistry and Physics. 91, 343347. 